\title{
Erratum to: Quality appraisal of clinical practice guidelines on glioma
}

\author{
Hongliang Tian • Yani Gou • Yawen Pan • Qiao Li • \\ Dang Wei • Zhenwei Wang • Xiaodong Niu • \\ Wentao Liang • Yinian Zhang
}

Published online: 24 September 2014

(C) Springer-Verlag Berlin Heidelberg 2014

\section{Erratum to: Neurosurgical Review}

DOI 10.1007/s10143-014-0569-z

The original version of this paper contained a mistake. Yinian Zhang should be the corresponding author and solely responsible for this article.

The online version of the original article can be found at http://dx.doi.org/ 10.1007/s10143-014-0569-z.

H. Tian

Jinling Hospital, Medical School of Nanjing University,

Nanjing 210002, China

Y. Gou $\cdot$ X. Niu $\cdot$ W. Liang

The Second Clinical Medical School of Lanzhou University,

Lanzhou 730000, China

Y. Pan $\cdot$ Q. Li $\cdot$ Y. Zhang $(\bowtie)$

Neurosurgery Department, The Second Hospital of Lanzhou

University, No. 82, Cui Ying Road, Lanzhou 730000, Gansu, China

e-mail: 2621069703@qq.com

D. Wei

Basic Medical School of Lanzhou University, Lanzhou 730000,

China

Z. Wang

The First Clinical Medical School of Lanzhou University,

Lanzhou 730000, China 